\title{
Family ENgagement in Intensive Care Environments (FENICE): A quasi-experimental study protocol
}

\author{
Matteo Danielis, ${ }^{1,2}$ Alvisa Palese, ${ }^{2}$ Stefano Terzoni, ${ }^{3}$ Anne Destrebecq ${ }^{4}$ \\ ${ }^{1}$ Department of Clinical Sciences and Community Health, University of Milan, Milan; ${ }^{2}$ Department of \\ Medical Sciences, University of Udine, Udine; ${ }^{3}$ School of Nursing, San Paolo Hospital, Milan; ${ }^{4}$ Department \\ of Biomedical Sciences for Health, University of Milan, Milan, Italy
}

\begin{abstract}
Even if health care professionals should take into account the active involvement of families in Intensive Care Units (ICUs), little research that investigates family member's contributions to care and includes its outcomes on both the family itself and the patient care, is available. The aim of this research project is to assess the effects of a family engagement program on family members' satisfaction and on patients' well-being and quality of life. A quasiexperimental study with two non-randomized groups (94 per each group) will be performed in two general ICUs of an Academic Italian Hospital. The intervention will be carried out in a single ICU and it consists of family members' involvement in the care of their loved ones by means of bed physical exercises and simple care tasks. Participants will be evaluated through manifold outcomes: family satisfaction, assessed with the FS-ICU tool within 48 hours after ICU discharge; patients' sense of well-being, measured with a visual analogue scale within 30 minutes after the time period of a visit; and the quality of life, investigated with the SF12 questionnaire within the first 48 hours after admission to ICU,
\end{abstract}

Correspondence: Matteo Danielis, Department of Clinical Sciences and Community Health, University of Milan, Via Vanzetti, 5 - 20133, Milan, Italy.

E-mail: matteo.danielis@unimi.it

Key Words: family engagement; intensive care unit; critical care; patient and family outcomes; experimental study.

Ethical Committee: This protocol was approved by the Regional Ethics Committee of the Friuli Venezia Giulia, Italy (CEUR-2020-Sper-012).

Trial registration: ClinicalTrials.gov, Identifier: NCT04311190. Registered on March 17, 2020. Available from: https://clinicaltrials.gov/ ct $2 /$ show $/$ NCT04311190?term $=$ fenice $\&$ draw $=2 \&$ rank $=1$.

Conflict of interest: No one. This work was not supported by any grant. Ethics approval and consent to participate: The manuscript does not contain any individual person's data in any form.

Received for publication: 19 March 2020.

Accepted for publication: 27 May 2020.

This work is licensed under a Creative Commons Attribution 4.0 License (by-nc 4.0).

(C) Copyright: the Author(s), 2020

Licensee PAGEPress, Italy

Emergency Care Journal 2020; 16:8972

doi:10.4081/ecj.2020.8972 at three and six months after ICU discharge by telephone. From July 2020, 188 subjects are going to be enrolled throughout the year. The latest data will be collected in December 2021 to allow the completion of the follow-ups of all participants. First, this study constitutes a significant step in a research agenda aimed at deepening the nursing sensitive outcomes in ICUs and the quality of hospital care. Secondly, the results of this study might have the potential to provide a better understanding of how families may modify patients' outcomes and whether family members would benefit from an involvement program.

\section{Introduction}

The well-being of patients and their relatives is considered fundamental in the care of critically ill adult patients and one of the top five research priorities among intensive care nurses. ${ }^{1}$ The concept of "family engagement" in Intensive Care Unit (ICU) has expanded to the point where Health Care Professionals (HCPs) should now consider the role of the family as no longer of passive consultation, but rather of active involvement. ${ }^{2}$

A recent scoping review has investigated the extent of literature on patient and family involvement in ICUs between 2003 and 2014. ${ }^{3}$ The authors identified five components of family participation, that focused on involvement as: i) being present; ii) receiving care and meeting needs; iii) communicating and receiving information; iv) participating in the decision-making process; and v) contributing to care. ${ }^{3}$ A more recent clinical review in 2018 has summarized the concept of "engagement" by defining it as a mechanism to take action with people and to share with them information and decisions, and a way to achieve patient- and family-centered care.$^{4}$ In addition, authors designated as "family" each family member, close relative, and caregiver with whom the patient spent most of the time. ${ }^{4}$

Where "family involvement" has been documented, the contributions have primarily considered family's attendance in ICU rounds, ${ }^{5-7}$ followed by the participation in fundamental care, such as bathing and massaging, ${ }^{8}$ in psychological patients' care, ${ }^{9}$ in ventilator weaning programs, ${ }^{10}$ and in research experiences. ${ }^{11}$

Regarding the outcomes, the family members' involvement in the ICU patient care seems to improve patients' psychological recovery, well-being, and satisfaction levels, ${ }^{12}$ while their posttraumatic disorders and depression were found to be reduced. ${ }^{13}$ Recently, family engagement in professional care has been recognized as a Nursing Sensitive Outcome (NSO), ${ }^{14,15}$ that is a patient or caregiver's state, behavior, or perception associated to nursing interventions. ${ }^{16}$ Accordingly, assessing family members' satisfaction should be considered a way to improve the quality of critical care provided to families of patients in ICU environments. ${ }^{17}$

To date, little research investigating family members' contributions to care in ICUs, including its outcomes on family itself and on 
patient care, is available. ${ }^{3}$ We hypothesized that engaging families in the care of critically ill patients could improve outcome both at family and at patient levels. Thus, the aim of this project is to assess the effects of a family engagement program on family members' satisfaction and on patients' well-being and Quality of Life (QoL).

\section{Materials and Methods}

\section{Research hypothesis and expected outcomes}

i) At family level: Families of critically ill patients in the family engagement group will show a higher general satisfaction as compared to those families in the control group.

ii) At patient level: The family-involved patients' group will show a) an increased sense of well-being after the daily visiting period, and b) a better QoL after ICU discharge (three and six months) as compared than the control group.

\section{Study Design}

A quasi-experimental study with two non-randomized groups will be performed by following the CONSORT (CONsolidated Standards Of Reporting Trials) guidelines. ${ }^{18}$ Figure 1 shows the CONSORT flow diagram corresponding to the present study.

\section{Setting}

This study will be conducted in two general ICUs of an Academic Hospital in the Northeast of Italy equipped with 900 beds. ICU A and B (beds = eight/each) provide care for both ventilated and non-ventilated patients and mixed medical, surgical, and trauma patients admitted directly through the Emergency Department (ED) or other hospital wards. Each ICU environment is a traditional open space, in which beds are separated by curtains. Staff is composed of Registered Nurses (RNs), with a nurse-topatient ratio of 1:2, Medical Doctors (MD) and Nursing Assistants (NAs). Generally, the average ICU Length Of Stay (LOS) is 5.0 days and the average ICU occupancy rate is $80.2 \%$.

\section{Sampling and sample}

This study will involve two groups of family members of patients who will be admitted to two ICUs. Specifically, in the intervention group, family members will be actively involved in the care of their relative; in the control group, patients will receive the usual care provided only by the nurses. Given the setting of the ICUs, with one open space shared by patients and visitors, participant randomization is not feasible. Moreover, to reduce the risk of inter-group contamination, ICU-A will be assigned to the intervention group and ICU-B to the control group.

A consecutive sampling method will be adopted to recruit the expected family members of adult patients ( $\geq 18$ years) in both the intervention and the control groups in the same period, from July 2020 to June 2021. Inclusion and exclusion criteria for the study population are shown in detail in Table 1. Patients' withdrawal criteria will be as follows: discharge from ICU, transfer to another hospital, or death. The withdrawal criterion for family members will be the unexpected suspension of daily visiting.

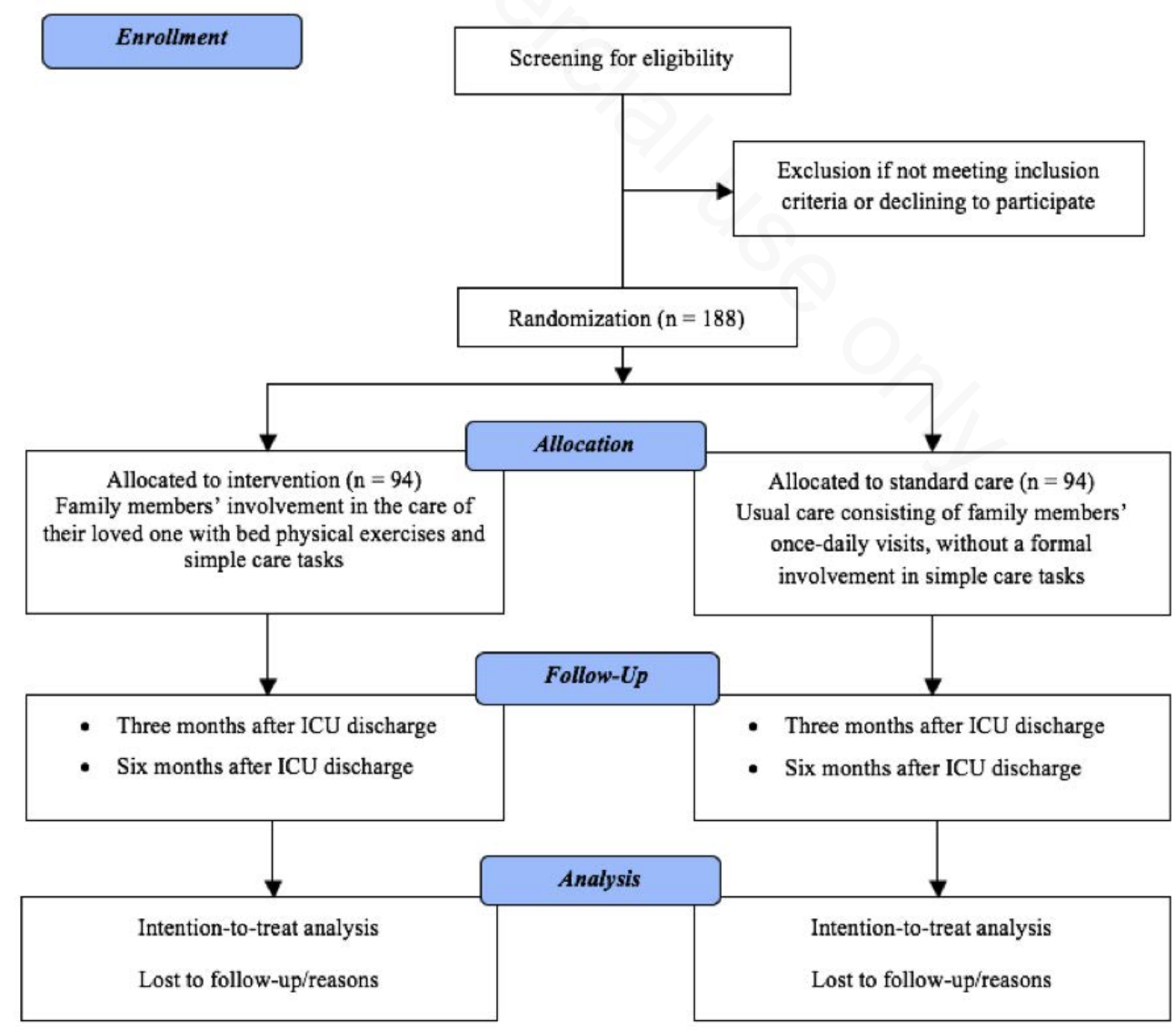

Figure 1. Flow chart of the study protocol. 


\section{Sample size calculation}

The sample size calculation is based on the target population of the study, which is composed of family members. Yet, no studies have evaluated the specific effect of the implementation of a family involvement program in ICUs on family's' perceived satisfaction. However, previous studies estimated the effect of structured communication and support programs on the satisfaction of family members of critically ill patients with the FS-ICU tool, ${ }^{19,20}$ in which the mean of FS-ICU total score before intervention was 55.3 $( \pm 10.3)$ and after intervention was $61.4( \pm 17.7)$. Based on this, a sample of 188 patients (94 per each group) is the adequate requirement for the present study. This will result in a mid-scale effect size of 0.41 , at a $5 \%$ significance level with a power of $80 \%$ and an allocation rate of $1: 1$. The sample size of this study was calculated with the software G*Power (version 3.1.9.4). ${ }^{21}$ Thus, each family member will be included together with his/her loved one who is being taken care for in ICU (94 per each group).

Despite light sedation being considered a routine therapy in the ICU settings of this study, a variety of reasons, including neurological, physiological, and communicative disabilities, can prevent patients' accurate estimation of their well-being by means of self- reported measures. This group of patients may include unconscious, deeply sedated and intubated patients, as well as those with a cognitive impairment or a head and maxillofacial trauma. For this reason, the researchers could not extend the sample size evaluation to the patient' well-being outcome.

\section{Intervention and control group}

The intervention will be carried out in a single ICU (ICU-A) and will engage family members in the care of their loved one with bed physical exercises and simple care tasks, as reported in Table 2. Trained nurses will provide families with written and verbal information about their involvement in the above-mentioned care activities (day 1: patient's admission to ICU) and will strictly supervise their engagement during the daily visiting hours (from day 2 to the transferring into another unit/ward). No intervention will be implemented without the approval of nurses and physicians who daily evaluate patients' conditions. Each session of family involvement will last for at least one hour per patient/day. In the case of signs of respiratory, hemodynamic or neurovegetative distress, the session will be immediately suspended by any HCP and the reasons of such decision will be appropriately communicated to the family member. The interven-

Table 1. Inclusion and exclusion criteria for patients and family members

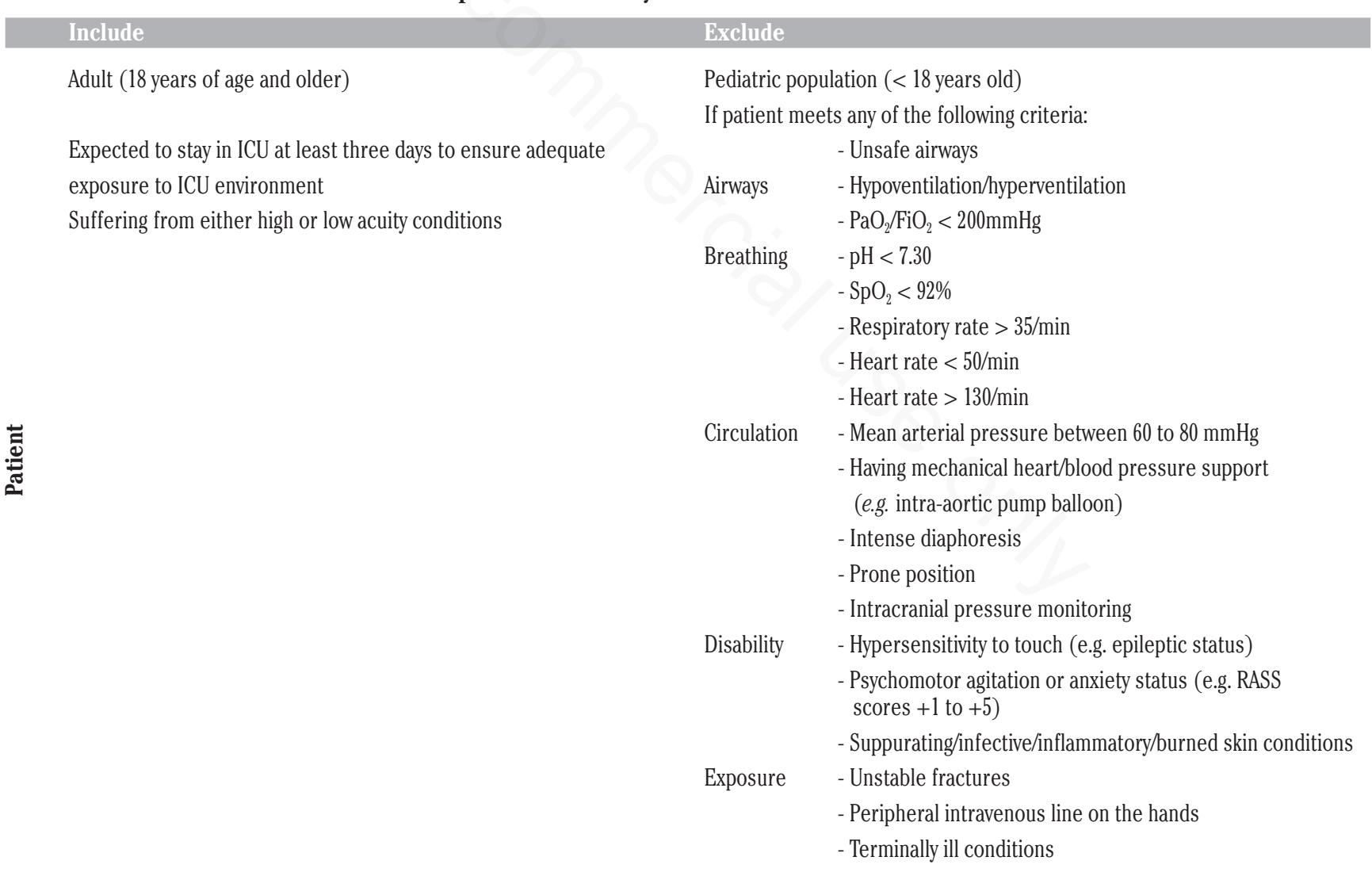

Adult (18 years of age and older)

Being the patient's spouse, or in a blood relation, or next of kin, and/or a person with whom the patient spends most of the time Willing to visit the patient daily

Willing to participate in the study
Pediatric population ( $<18$ years old)

Lack of propensity or interest in participating in the study

RASS $=$ Richmond Agitation Sedation Scale 
tion group will be formed of maximum eight patients, corresponding to the ICU-A bed availability. In the ICU-B, patients will receive the usual care consisting of a once-daily visit of at least one hour by family members without their formal involvement in simple care.

\section{Family members' recruitment and involvement}

Table 3 illustrates the recruitment and the involvement processes for family members.

\section{Data collection and measurements}

All data will be collected daily by two researchers (MD, AP), together with the nursing and medical staff of the Department of Anaesthesia and Intensive Care, and professionally trained medical and nursing students. Data collection will include both clinical information and paper-based questionnaires. The anonymized paper data will be stored by the researchers (see authors) of the present study.

\section{Sociodemographic and clinical data}

At the time of admission to ICU, within the routinely admission assessment, patients' demographics (age, gender), clinical characteristics (diagnosis, comorbidities, Glasgow Coma Scale [GCS] and Richmond Agitation-Sedation Scale [RASS] scores), anthropometric measures (weight and height, both required to calculate the Body Mass Index [BMI]), and previous habits (e.g., cigarette smoking) will be assessed. Simultaneously, the family member's information, including age, gender, education, relation to patient and profession, will be collected with an ad hoc form.

\section{Intervention}

Every day, each activity performed by the family member in the intervention group will be recorded in a pre-established form. The grid will report data, time, type of activities, and their duration (in minutes). In case of clinical deterioration during the intervention, this will be immediately stopped by the nurse and/or physician in charge of the patient's care. The intervention group will be formed of maximum eight patients, corresponding to the ICU-A bed availability.

\section{Outcomes}

At family level: Family satisfaction, assessed with the FS-ICU tool, ${ }^{22,23}$ will be self-reported by family members within 48 hours after a patient's discharge from ICU.

At patient level: i) A patient's sense of well-being will be measured with a visual analogue scale (VAS) within 30 minutes after the family involvement for the intervention group and in the same span of time after the ICU visiting hours for the control group; ${ }^{24}$ ii) The QoL will be investigated with the SF-1225 questionnaire within the first 48 hours after admission to assess preadmission QoL, ${ }^{26}$ at three and six months after ICU discharge by telephone (patient or family members).

\section{FS-ICU}

The FS-ICU questionnaire includes 24 items; 14 inquire about families' satisfaction with care and 10 explore their satisfaction with decision-making. ${ }^{22}$ All items are scored on a five-point Likert scale (from $1=$ very dissatisfied to $5=$ very satisfied) except one item which is dichotomous (yes/no). All questions included the

Table 2. Family involvement: care activities

\begin{tabular}{|c|c|}
\hline $\begin{array}{l}\text { High acuity patients } \\
\text { (when a patient is mechanically ventilated and under analgosedation) }\end{array}$ & $\begin{array}{l}\text { Low acuity patients } \\
\text { (when a patient is awake and collaborative) }\end{array}$ \\
\hline Open and close the fingers & Massages \\
\hline Open and close the toes & Arms and legs position changes \\
\hline Physical presence with touch at least 60 minutes/day & Washing face \\
\hline Hair brushing & Assisting with rehabilitation exercises \\
\hline Reading texts of interest to the patient (e.g., newspapers) & $\begin{array}{l}\text { Encouraging/assisting with eating and drinking under nurses' strict } \\
\text { supervision }\end{array}$ \\
\hline
\end{tabular}

Table 3. Details of the intervention protocol

$$
\text { Step } 1 \text { - Day one (patient's admission to ICU) }
$$

The researcher ${ }^{\mathrm{a}}$ verifies if the patient meets the inclusion criteria.

If yes, the researcher ${ }^{\mathrm{a}}$ proceeds with step 2.

\section{Step 2 - Day one (family members' first visit to the ICU)}

The researcher ${ }^{a}$ verifies if the family member meets the inclusion criteria.

If yes, the researchera provides information about the intervention (including how to talk to the patient, advice on where to sit or stand at the bedside and on how to open and close the interaction, as well as on the activities in which family members can be involved). The education session will last at least half an hour. Moreover, the researchera discusses the study details with the family, identifies with them a family member as a reference, and ascertains decisions regarding consent.

If the written consent is provided, data collection starts with step 3.

\section{Step 3 - Day two to transfer to ward (during the ICU-stay)}

The researchera actively seeks opportunities to promote the family access to the patient, maximizing the time available for the family to be with s/he and encouraging them to interact by talking and holding the patient's hand. In addition, the researcher ${ }^{\text {a }}$ promotes the implementation of the activities described in Table 2 by recording them in the ad hoc grid.

On daily basis, the researchera evaluates both the availability of the family member to participate, and the patient's clinical conditions before engaging the family member. Furthermore, the researcher ${ }^{\mathrm{a}}$ supervises all activities performed by the family member and provides feedback of encouragement.

ICU = Intensive Care Unit. a the authors and/or a member of the research team. 
"not applicable" optional response. Data will be collected from all participants via self-administered questionnaires (written).

\section{$S F-12$}

Originally developed from the Medical Outcomes Study (MOS) 36-item Short-Form Health Survey (SF-36), the SF-12 tool consists of 12 questions and measures eight domains: physical functioning, role-physical, bodily pain, general health, vitality, social functioning, role-emotional, and mental health. ${ }^{25,27}$ A score ranging from 0 (indicating the worse health status) to 100 (the best health status) is assigned to each domain. Domain scores can be summarized into a Physical Component Score (PCS) and Mental Component Score (MCS). Data will be collected on all participants via a combination of self-administered questionnaires (written) during the ICU-stay and telephone interviews post-discharge.

\section{Data analysis}

Collected data will be entered into a Microsoft Excel® ${ }^{\circledR}$ worksheet. All statistical analysis will be performed using the SPSS software version 25 (SPSS Institute Inc., USA) and the statistical significance set at $\mathrm{p}<0.05$. Continuous variables (e.g. age, LOS) will be displayed as mean and Standard Deviation (SD) if normally distributed or median and quartiles otherwise. Normality will be assessed by using the Kolmogorov-Smirnov test. Nominal variables (e.g. gender, reason of admission) will be calculated as absolute numbers and percentage. Student's unpaired t-test will be used to analyze comparisons between means; Blom's transformation will be used if normality test fails. Categorical variables will be analyzed using the chi-squared test for independence. A continuity-corrected chi-squared statistic or Fisher's exact test to evaluate the null hypothesis of this study in case of expected frequencies $<5$ in contingency tables will be applied. Moreover, the scores of FS-ICU and SF-12 obtained from the two groups will be examine using the analysis of covariance (ANCOVA).

Once half of the sample is reached (47 per each group), an ad interim analysis will be performed to assess i) whether the intervention is feasible in terms of family members acceptability and willingness to participate in the study, and ii) whether a statistically significant difference regarding family satisfaction between groups is already noticeable ( $\mathrm{p}$-value set to 0.05 ). According to the results (e.g., study dropout rates and consent refusal, treatment difference larger or smaller than expected), the intervention and/or sample size calculation will be changed, and a new research protocol will be developed. However, this study has not stopping rules.

\section{Ethical considerations}

The current study will be conducted according to the criteria set by the declaration of Helsinki; each patient legally authorizes a representative and/or a family member will provide written informed consent for all study procedures. Family members will be informed about withdrawing from the study at any time without affecting the care provided. The participants' privacy and personal information will be protected, and data will be anonymously analyzed. In addition, both ICUs are equipped to ensure patients' privacy during the visiting and intervention time (e.g. large spaces, beds separated by curtains). Lastly, this protocol was approved by the Regional Ethics Committee of the Friuli Venezia Giulia (CEUR-2020-Sper-012).

\section{Results}

Beginning from July 2020, 188 subjects are going to be enrolled throughout the year. The latest data will be collected in December 2021 to allow the completion of the follow-ups of all participants.

\section{Discussion}

To our best knowledge, very few works ${ }^{19,20}$ have studied the relationship between engaging families' interventions and NSOs in ICUs. This protocol constitutes a significant step in a research agenda aimed at deepening the NSOs' reliability across the ICUs and useful to proxy monitoring the quality of hospital care. The results of this study might have the potential to provide additional evidence for family participation in bedside care in ICUs, and a better understanding of how family members and critically ill patients may benefit from the involvement itself.

Regarding dissemination, the results of the present study will be firstly presented and discussed at professional meetings by conducting a stakeholders' consultation. Then, research findings will be disseminated in scientific journals and at national and international conferences.

\section{Conclusions}

Despite international bodies prioritizing the well-being of families and patients in ICU, current literature lacks practical underpinnings and examples to promote the involvement of family in the care of the critically ill patients. This study protocol aimed at examining the effect of a family engagement program on NSOs at both family- and patient-level. The results may provide valuable insight into the quality of care delivered. ICU nurses and physicians need to become convinced that families can be routinely involved in care activities; family engagement can optimize patients' outcomes including well-being and quality of life, and increase family satisfaction with care in ICU.

\section{References}

1. Blackwood B, Albarran JW, Latour JM. Research priorities of adult intensive care nurses in 20 European countries: a Delphi study. J Adv Nurs 2011;67:550-62.

2. Haines KJ. Engaging Families in Rehabilitation of People Who Are Critically Ill: An Underutilized Resource. Phys Ther 2018;98:737-44.

3. Olding M, McMillan SE, Reeves S, et al. Patient and family involvement in adult critical and intensive care settings: a scoping review. Health Expect 2016;19:1183-202.

4. Burns KEA, Misak C, Herridge M, et al. Patient and Family Engagement in the ICU Untapped Opportunities and Underrecognized Challenges. Am J Respir Crit Care Med 2018;198:310-9.

5. Au SS, des Ordons ALR, Leigh JP, et al. A Multicenter Observational Study of Family Participation in ICU Rounds. Crit Care Med 2018;46:1255-62.

6. Stelson EA, Carr BG, Golden KE, et al. Perceptions of family participation in intensive care unit rounds and telemedicine: a qualitative assessment. Am J Crit Care 2016;25:440-7.

7. Stickney CA, Ziniel SI, Brett MS, Truog RD. Family Participation during Intensive Care Unit Rounds: Attitudes and Experiences of Parents and Healthcare Providers in a Tertiary 
Pediatric Intensive Care Unit. J Pediatr 2014;165:1245-51.

8. Mitchell M, Chaboyer W, Burmeister E, Foster M. Positive effects of a nursing intervention on family-centered care in adult critical care. Am J Crit Care 2009;18:543-52.

9. Black P, Boore JRP, Parahoo K. The effect of nurse-facilitated family participation in the psychological care of the critically ill patient. J Adv Nurs 2011;67:1091-101.

10. Burns KEA, Devlin JW, Hill NS. Patient and Family Engagement in Designing and Implementing a Weaning Trial A Novel Research Paradigm in Critical Care. Chest 2017; 152:707-11.

11. Dotolo D, Nielsen EL, Curtis JR, Engelberg RA. Strategies for Enhancing Family Participation in Research in the ICU: Findings From a Qualitative Study. J Pain Symptom Manage 2017;54:226-30.e1.

12. Huffines M, Johnson KL, Naranjo LLS, et al. Improving Family Satisfaction and Participation in Decision Making in an Intensive Care Unit. Crit Care Nurse 2013;33:56-68.

13. Oczkowski SJW, Mazzetti I, Cupido C, Fox-Robichaud AE. The offering of family presence during resuscitation: a systematic review and meta-analysis. J Int Care 2015;3:11.

14. Minton C, Batten L, Huntington A. The impact of a prolonged stay in the ICU on patients' fundamental care needs. J Clin Nurs 2018;27:2300-10.

15. Castellan C, Sluga S, Spina E, Sanson G. Nursing diagnoses, outcomes and interventions as measures of patient complexity and nursing care requirement in Intensive Care Unit. J Adv Nurs 2016;72:1273-86.

16. Maas ML, Johnson M, Moorhead S. Classifying nursing-sensitive patient outcomes. Image J Nurs Sch 1996;28:295-301.

17. Frivold G, Slettebo A, Heyland DK, Dale B. Family members' satisfaction with care and decision-making inintensive care units and post-stay follow-up needs-a cross-sectional survey study. Nurs Open 2018;5:6-14.
18. Schulz KF, Altman DG, Moher D, Grp C. CONSORT 2010 Statement: updated guidelines for reporting parallel group randomised trials. Trials 2010;11:8.

19. Abvali A, Peyrovi H, Moradi-Moghaddam O, Gohari M. Effect of Support Program on Satisfaction of Family Members of ICU Patients. J Client Nurs Care 2015;1:29-36.

20. Othman H, Subramanian P, Ali NAM, et al. The Effect of Information Booklets on Family Members' Satisfaction with Decision Making in an Intensive Care Unit of Malaysia. J Young Pharm 2016;8:128-32.

21. Faul F, Erdfelder E, Lang AG, Buchner A. G*Power 3: A flexible statistical power analysis program for the social, behavioral, and biomedical sciences. Behav Res Met 2007;39:17591.

22. Wall RJ, Engelberg RA, Downey L, et al. Refinement, scoring, and validation of the Family Satisfaction in the Intensive Care Unit (FS-ICU) survey. Crit Care Med 2007;35:271-9.

23. Heyland DK, Tranmer JE, Kingston Gen Hosp ICURW. Measuring family satisfaction with care in the intensive care unit: The development of a questionnaire and preliminary results. J Crit Care 2001;16:142-9.

24. Lolaty HA, Bagheri-Nesami M, Shorofi SA, et al. The effects of family-friend visits on anxiety, physiological indices and well-being of MI patients admitted to a coronary care unit. Complement Ther Clin Pract 2014;20:147-51.

25. Apolone G, Mosconi P. The Italian SF-36 Health Survey: Translation, validation and norming. J Clin Epidemiol 1998;51:1025-36.

26. Hofhuis JG, Spronk PE, Van Stel HF, et al. Quality of life before intensive care unit admission is a predictor of survival. Crit Care 2007;11:7.

27. Ware JE, Kosinski M, Keller SD. A 12-item short-form health survey - Construction of scales and preliminary tests of reliability and validity. Medical Care 1996;34:220-33. 